Aus der Universitätskinderklinik in Berlin. (Direktor: Geheimrat Czerny.)

\title{
Zur Prophylaxe der Kindertuberkulose im Kriege.
}

Von Priv.-Doz. Dr. H. Kleinschmidt.

Es ist bekannt, daß die Tuberkulosemorbidität und -mortalität infolge des Krieges wiederum eine Zunahme erfahren hat, nachdem in den letztvergangenen Jahren eine dauernde $\mathrm{Ab}$ nahme der Sterbeziffer festgestellt werden konnte. Nicht zuletzt müssen wir dies von der Kindertuberkulose sagen, die schon vor dem Kriege an der allgemeinen Abnahme nicht, teilgenommen hat $\left(\mathrm{Beh}_{\mathrm{a}} \mathrm{a}^{\mathrm{I}}\right)$ ). Ein großer Teil der Kindertuberkulosen verläuft anfangs so schleichend und unter so unbedeutenden Erscheinungen, daß erst in einem späten Stadium das Leiden erkannt wird und oft erst erkannt werden kann. Therapeutische Maßnahmen sind zu dieser Zeit vollkommen erfolglos und würden es selbst sein, wenn nennenswerte Fortschritte auf dem Gebiete der Tuberkulosetherapie erreicht werden sollten. Mit gebieterischer Notwendigkeit werden wir daher vor die Aufgabe einer wirksamen Tuberkuloseprophylaxe gestellt. Das ist schon unzählige Male ausgesprochen und in mannigfaltigster Weise erläutert worden, und doch sind wir von dem Ziele so weit entfernt, daß es nur in nebelhafter Ferne geahnt werden kann.

Die tägliche Beschäftigung mit zahllosen verzweifelten Tuberkulosefällen, die in unsere Poliklinik und Klinik gebracht werden, veranlaßt mich, noch einmal'2) ${ }^{3}$ ) hierzu das Wort zu ergreifen, um auf diejenigen Maßnahmen aufmerksam zu machen, die im Augenblick sich als besonders dringend erweisen.

Einer der wichtigsten Faktoren für die Entwicklung und den Verlauf einer Erkrankung an Tuberkulose ist die Art der Ernährung. Wir wissen, daß insbesondere die einseitige Kohlehydraternährung die Tuberkulose ungünstig beeinflußt, und es ist daher folgerichtig, wenn wir die augenblickliche Häufung der Tuberkuloseerkrankungen mit der uns aufgezwungenen Ernährungsform in Verbindung bringen. Dieser Faktor allein kann jedoch nicht maßgebend sein. Denn wir ahen einerseits schon zahlreiche Tuberkuloseerkrankungen im Säuglingsalter, dessen Ernährungsformen nicht so erheblich ge-

1) 11. Internationale Tuberkulosekonferenz, Berlin 1913. - ') Die Therapie des praktischen Arztes. Herausgegeben von E. Müller. Berlin 1914. - 3) Der Arzt als Erzieher, 12. Jahrg. 1916 H. 6. 
ändert worden sind, und wissen anderseits aus der täglichen Erfahrung, daß auch das ältere Kind vielfach weniger unter der einseitigen Ernährung zu leiden hat als der Erwachsene, der gerne zugunsten seiner Kinder auf manches Verzicht leistet. Immerhin ist der Ernährungsfaktor von zweifellos größter Wichtigkeit, und man sollte sich bemühen, seine Bedeutung nach Möglichkeit einzuschränken. Leider ist dies nach Lage der Dinge nur schwer zu erreichen. Wir haben durch reichliche Verordnungen von Lebertran in geeigneten Fällen die Fettarmut der Nahrung auszugleichen versucht, sind uns aber bewußt, daß auch dieses Korrigens nur in beschränktem Umfange zur Verfügung steht und sein Preis erheblich emporgeschnellt ist.

Um so mehr Interesse verdient eine Maßnahme auf diätetischem Gebiete, die sich jederzeit und überall mit Leichtigkeit durchführen läßt, nämlich die Warnung vor dem Genusse roher Milch. Die Untersuchungen von Hart und Rabinowitsch ${ }^{1}$ ) haben es wahrscheinlich gemacht, daß die augenblickliche Tuberkulosehäufung zum Teil auf der Zunahme boviner Infektionen beruht, und unsere eigenen Beobachtungen sprechen in gleichem Sinne, da sie ein besonders gehäuftes Auftreten von abdominalen Tuberkulosen vielfach mit entsprechender Anamnese anzeigen. Es ist ja auch ganz verständlich, daß bei der verminderten tierärztlichen Kontrolle und dem vielfachen Fehlen sachkundiger Stallschweizer die Rindertuberkulose sich leichter der Feststellung entzieht als sonst.

Wie weit wir zurzeit von einer allgemeinen Warnung vor dem Genusse ungekochter Milch entfernt sind, lehrt die bedauerliche Tatsache, daß noch immer von vielen Seiten die Darreichung roher Milch an Kinder ärztlich verordnet wird. Auf diese Weise muß natürlich im Volke die Meinung aufrecht erhalten bleiben, daß ihren Kindern eine besondere Wohltat erwiesen wird, wenn sie mit roher Milch ernährt werden. In Wirklichkeit entspricht jedoch die ärztliche Verordnung von roher Milch keineswegs dem Stande der Wissenschaft. Durch umfangreiche Untersuchungen von Czerny, Finkelstein v. a. ist dargetan worden, daß die Verwendung von roher Milch bei den Ernährungsstörungen der Säuglinge keine Vorteile gegenüber der gekochten Milch mit sich bringt, desgleichen wissen wir heute, daß die Rachitis weder durch rohe Milch verhütet noch geheilt werden kann, ${ }^{2}$ ) und schließlich haben schon vor zehn Jahren Czerny und $\mathrm{Keller}^{3}$ ) berichtet, daß die Forderung der Verwendung roher Milch als therapeutisches Mittel bei der Barlowschen Krankheit ihren eigenen Erfahrungen und denen mancher anderen Aerzte widerspricht. In der Tat kann man sich immer wieder davon überzeugen, $\mathrm{da} ß$ ebenso gut Heilung mit einer kurz abgekochten Milch aus einer anderen Stallung unter Zugabe von Gemüse und Obst erzielt werden kann. Somit bestehen die früher vielfach gelehrten und heute noch in manchen Lehrbüchern festgehaltenen Indikationen zur Ernährung mit roher Milch bei keiner Erkrankung des Kindes mehr, und man sollte unbedingt und zu allen Zeiten auf dem Abkochen (oder zum mindesten Pasteurisieren) jeder Tiermilch bestehen, die zur menschlichen Ernährung benutzt wird.

Weit häufiger aber und deshalb auch wichtiger als die Infektion mit bovinen ist diejenige mit humanen Tuberkelbazillen. Den hustenden Phthisiker gilt es von dem Kinde fernzuhalten, ganz besonders aber. von dem Kinde der ersten Lebensjahre, bei dem die Neigung zur Generalisierung der Tuberkulose so ungeheuer groß ist. Wie weit wir in diesem Punkte von einem Idealzustand entfernt sind, wird jeder zugeben, der weiß, daß in unzähligen Fällen dem erwachsenen Tuberkulösen die Art seiner Erkrankung vom Arzte verschwiegen oder umschrieben, geschweige denn die Ansteckungsmöglichkeit auseinandergesetzt wird. Dies täglich erfahren zu müssen, ist eine bittere Enttäuschung für den Pädiater. Aber schon so mancher Mahnruf, wie der $\left.\mathrm{Czernys}^{4}\right)$ : , alle die wahre Natur des Leidens verbergenden Diagnosen fallen zu lassen und jedesmal die Krankheit mit ihrem richtigen Namen zu bezeichnen", ist ungehört verklungen. Man spricht unbeirrt weiter von Lungen-

1) Zschr. f. Tbc. 27. 1917. - 2) Janet, E. Lane-Claypon, Ergebnisse der inn. Medizin u. Kinderhlk. 10. $-{ }^{3}$ ) Des Kindes Ernährung, Ernährungsstörungen und Ernährungstherapie, Wien 1907. - $\left.{ }^{4}\right)$ B. kl. W. $1990 \mathrm{Nr} .46$. spitzenkatarrh, Lungenkatarrh, Lungenschwäche und Skrofulose in Krankheitsfällen, wo einwandfrei festgestellte Tuberkulose vorliegt. Man glaubt damit den Erwachsenen zu schonen und schädigt das Kind. Es ist aber nicht einmal richtig, daß die Aufklärung des Erwachsenen über die Natur seiner Erkrankung für ihn nachteilig ist, nachdem er jederzeit und überall hören kann, daß Tuberkulose eine heilbare Krankheit ist.

Die Tätigkeit des Arztes ist nicht mit der Erkennung und Behandlung einer Erkrankung beendet. Zum mindesten nicht bei einer Infektionskrankheit. Tatsächlich geht sie auch bei den akuten Infektionskrankheiten in der Regel zum Wohle der Umgebung des Kranken weiter. Nur bei der Tuberkulose sehen wir häufige Ausnahmen von dieser Regel. In unserer Klinik und Poliklinik bemühen wir uns, in jedem Tuberkulosefall, der uns zu Gesicht kommt, die Infektionsquelle aufzudecken, um sie für das Kind selbst, seine Geschwister und sonstigen Mitbewohner auszuschalten. Wissen wir doch, daß die Tuberkuloseimmunität, wie sie fast jeder Mensch in mehr oder weniger starkem Maße erwirbt, erst im Laufe von Jahren eine für das Individaum nützliche Höhe erreichen kann. Wir müssen also bei dem eben infizierten Kinde unbedingt Reinfektionen verhüten, wenn wir die Abheilung der Erkrankung unterstützen wollen. Nebenbei aber treiben wir mit der Feststellung der Infektionsquelle eine zielbewußte Prophylaxe durch Aufklärung des Publikums und Verhütung von Infektionen bei anderen Kindern der Familie. Es bedarf vielfach sehr eingehender Nachfragen, um die Infektionsquelle des Kindes ausfindig zu machen; aber wenn man sich darum bemüht, gelingt es immerhin in einem recht beträchtlichen Prozentsatz, zumal bei jüngeren Kindern. Erhält man nicht sogleich Auskunft, so genügt es oft genug, den Angehörigen auf das bestimmteste $\mathrm{zu}$ sagen, daß das Kind mit einem Tuberkulosekranken vor kürzerer oder längerer Zeit in Berührung gekommen sein muß und daß sie die Pflicht haben, diesen in ihrer Umgebung festzustellen. Die daraus sich ergebenden Nachforschungen bringen dann vielmals noch genügende Aufklärung. Ich erinnere nur daran, daß Eltern oder Pflegepersonen nicht selten in Unkenntnis ihrer eigenen Erkrankung diese au. das Kind übertragen.

Bei solchen Nachfragen nun haben wir in der letzten Zeit Beobachtungen gemacht, die uns für die Häufung der Kindertuberkulose von ausschlaggebender Wichtigkeit zu sein scheinen. Die augenblicklichen sozialen Verhältnisse zwingen viele Eltern oder alleinstehende Mütter, ihre Kinder in fremde Pflege zu geben, in einem Maße, wie es zu anderer Zeit niemals vorkam. Nur ein Teil der Kinder wird in Horten, Krippen ünd sonstigen Anstalten untergebracht, die anderen befinden sich während längerer oder kürzerer Zeit bei Verwandten, Freunden oder auch in nicht näher bekannten Familien bald auf dem Lande, bald in der Stadt. So viele Vorteile diese Einzelpflege der Kinder im Gegensatz zum Anstaltsaufenthalt bietet, gerade hier ergibt sich vielfach für die Kinder die Gelegenheit zur Infektion mit Tuberkulose, die sie im elterlichen Hausstand bis dahin nicht besaßen und voraussichtlich auch weiterhin nicht gehabt hätten. Hier wird der Keim zu der schweren und nur allzu oft tödlichen Krankheit gelegt. Beispiele mögen dies erläutern:

Fall 1. Irene D., 3 Jahre alt. Eltern gesund. Vater im Felde. Mutter arbeitet außer dem Hause und hat daher das Kind von August 1915 bis April 1916 bei einer Tante untergebracht. Diese ist vor wenigen Tagen in die Tuberkulosebaracke der Charité eingeliefert worden. April 1916 stellen wir bei dem Kinde eine ausgedehnte rechtseitige Lungen. tuberkulose fest und sind zur Pneumothoraxbehandlung gezwungen.

Fall 2. Fritz J., 11/2 Jahre alt. Eltern gesund. Einziges Kind. Wenn die Mutter zur Arbeit ging, hat oft eine Nachbarin das Kind gewartet, die Januar 1916 an Lungentuberkulose gestorben ist. März 1916 wird das Kind mit ansgedehnter rechtseitiger Lungentuberkulose und kleinem pleuritischen Erguß bei uns vorgestellt.

Fall 3. Hilde K., 4 Jahre alt. Eltern und die Schwester gesund. Das Kind wird April bis Oktober 1916, um es besser zu pflegen, zu einer Tante aufs Land geschickt. Diese leidet angeblich an "Asthma". Die rechte Lunge soll fast fort sein. März 1917 kommt das Kind mit ausgedehnter rechtseitiger Hilustuberkulose in unsere Poliklinik.

Fall 4. Ernst Th., 6 Jahre ult. Eltern und Geschwister gesund. Eine Nachbarin, die die Kinder öfter beaufsichtigte, wenn die Mutter aus dem Hause ging, ist März 1916 an Lungentuberkulose in der Cha- 
rité gestorben. Januar 1917 erfolgt die Einlieferung des Kindes in die Klinik wegen Meningitis tuberculosa.

Diese Beispiele, deren Zahl sich leicht vermehren ließe, mögen genügen, um zu zeigen, auf welche Weise und aus welchem Grunde heutzutage vielfach die Tuberkulose auf Kinder bis dahin tuberkulosefreier Familien übertragen wird. Sie scheinen mir im Augenblick besonders beachtenswert, wo man sich bemüht, durch Verschickung zahlreicher Stadtkinder aufs Land den Gesundheitszustand unseres Nachwuchses zu heben. Es ist dringend geboten, darauf zu achten, daß die Kinder in eine sicher tuberkulosefreie Umgebung gelangen, denn sonst wird das Gegenteil des Erstrebten erreicht. Natürlich ist es unmöglich, die übermäßig beschäftigten Landärzte auch noch zu regelmäßigen Untersuchungen in Familien heranzuziehen, die Kinder aus der Stadt bei sich aufnehmen wollen. Aber es würde schon unendlich viel erreicht sein, wenn jeder Arzt es sich zur Pflicht machte, alle Lungentuberkulösen, die in seine Behandlung gelangen, ernstlich darauf hinzuweisen, daß ihre Erkrankung ansteckend ist und a $\mathrm{u}$ Berordentlich leicht besonders a uf Kinder übertragen werden kann. Das ist mindestens ebenso wichtig, wie die Behandlung des Tuberkulösen selbst. Erfreulicherweise befindet sich die weitaus größte Zahl der aufs Land verschickten Kinder bereits im Schulalter, in dem die Ansteckung bekanntlich weniger leicht $\mathrm{zu}$ so ernsten Erkrankungen führt wie im ersten Kindesalter.

Eine weitere Folgeerscheinung des Krieges und der durch ihn geänderten Lebensbedingungen in weiten Kreisen der Bevölkerung, die sich unmittelbar an die eben geschilderten Verhältnisse anschließt, ist die Einschränkung der Wohnungen und die verminderte Wohnungshygiene. Wir stellten dementsprechend mehrfach Infektionen der Kinder durch Aftermieter oder kranke Verwandte fest, die aus pekuniären Gründen die Wohnung mit der Familie teilten. Ferner wurden uns wiederholt uneheliche Kinder des ersten und zweiten Lebensjahres mit Tuberkulose überwiesen, die bei Haltefrauen untergebracht waren. Die genauere Nachforschung ergab, daß die Kinder sich ihre Infektion in der Pflegestelle selbst geholt hatten, ein Beweis dafür, daß die Kontrolle der Pflegestellen zurzeit nicht genügend durchgeführt wird. Ich kann dies alles nur ganz kurz erwähnen, möchte aber zum Schluß noch etwas eingehender eine Infektionsquelle besprechen, die an Häufigkeit kaum hinter den früher genannten zurücktritt und nicht übermäßig schwer beeinflußbar erscheint. Ich meine die Infektion der Kinder durch vom Militär entlassene Tuberkulöse. In der ersten Kriegszeit beobachteten wir ein gehäuftes Auftreten von epidemischer Genickstarre bei Kindern, und ich habe an anderer Stelle ${ }^{1}$ ) darauf hingewiesen, daß diese Erkrankungen sich auffallend oft an einen Urlauberbesuch anschlossen; es folgten die gleichen Erfahrungen bei der Ruhr, jetzt aber sind diese Krankheiten völlig zurückgetreten hinter der größten Volksseuche, der Tuberkulose. Der Heeresdienst hat manche vollkommen latente Tuberkuloseinfektion manifest werden lassen, hat manche leichte und nicht ansteckungsfähige Tuberkuloseerkrankung schnell zur weiteren Verbreitung geführt. In zahlreichen Fällen sind unter dem Einfluß der Kriegsstrapazen Erkrankungen aufgetreten, wie sie unter den Lebensbedingungen des Friedens sich in einem weit geringeren Prozentsatz entwickelt haben würden. In die Heimat zurückgekehrt, müssen die so Erkrankten eine entsprechend größere Verbreitung der Tuberkulose unter den Kindern herbeiführen, und wir sehen zurzeit diese Folgeerscheinung erstmals vor uns. Auch hier können einige Beispiele zur Erläuterung dienen:

Fall 1. Rudolf G., 10 Monate alt, uneheliches Kind gesunder Mutter, wohnt bei der Großmutter, wo sich ein entfernter Verwandter vom 1. bis 16. Dezember 1916 aufhielt. Dieser kam unmittelbar aus einer Lungenheilstätte, wohin er vom Militär aus geschickt worden war. Am 16. Dezember wurde er in ein hiesiges Krankenhaus überführt, wo er am folgenden Tage an seiner Lungentuberkulose verstarb. April 1917 wird uns das Kind zugeführt mit der Angabe, daß es seit acht Tagen krank sei. Diagnose: Meningitis tuberculosa.

Fall 2. Kurt S., 1 Jahr 8 Monate alt, erstes Kind. Eltern bisher gesund. Vater von August bis November 1914 im Felde, dann lange
Zeit in verschiedenen Lazaretten, Mitte März 1916 mit starkem Husten und Auswurf nach Hause entlassen. Hier hütete er, während die Mutter zur Arbeit ging, drei Monate hindurch das Kind. Tod an Lungentuberkulose und Pleuritis am 28. Juni 1916 in einem Lazarett, in das er wenige Tage zuvor (23. Juni) gebracht worden war. Im Dezember 1916 wird das Kind mit hochfieberhafter Hilustuberkulose in unserer Poliklinik vorgestellt.

Fall 3. Erna G., $1^{3 / 4}$ Jahre alt. Mutter und zwei ältere Geschwister gesund. Vater früher gesund, im Felde an Lungentuberkulose erkrankt, wurde nach 1/2 jährigem Lazarettaufenthalt Juni 1916 nach Hause entlassen. Tod an kavernöser Lungen-, Kehlkopf- und Darmtuberkulose am 28. Februar 1917 in der Kgl. Charité, wo er am 26. Februar 1917 aufgenommen worden war. November 1916 sucht die Mutter mit dem Kinde wegen ronjunctivitis und Keratitis ekzematosa die Poliklinik auf, Mai 1917 stellt sie das Kind wiederum vor, diesmal mit Unterhaut. zellgewebstuberkulose.

Fall 4. Siegfried S., 1 Jahr alt. Mutter und drei Geschwister zwischen 8 und 12 Jahren gesund. Vater früher gesund, war 7 Monate im Felde, erkrankte dort an Iungentuberkulose und war dieserhalb bis Ende November 1915 im Lazarett bzw. in einer Erholungsstätte. Als dienstuntauglich nach Hause entlassen, blieb er zunächst ohne Beschäftigung, arbeitete vorübergehend, mußte aber wiederholt das Krankenhaus aufsuchen. Tod am 29. April 1917. Das Kind wird uns am 16. April 1917 mit einer schweren beiderseitigen Lunı́ntuberkulose überwiesen.

Welche Stellung soll man zu solchen Beobachtungen einnehmen? Für die militärärztliche Beurteilung der Lungentuberkulose werden zurzeit folgende Gesichtspunkte aufgestellt ${ }^{1}$ ): 1. Fälle mit Tendenz zur Ausheilung sind einer Lungenheilstätte zu überweisen oder, wenn erst nach langer Zeit Wiederherstellung der Dienstfähigkeit zu erwarten ist, als zeitig dienstuntauglich nach Hause zu entlassen. 2. Stationär bleibende Fälle sind für arbeitsverwendungsfähig zu erklären oder, besser, als dauernd untauglich zu bezeichnen. 3. Alle aktiven fortschreitenden Tuberkulosen sind als dauernd dienstuntauglich zu entlassen. Eine Zurückhaltung insbesondere dieser letzten hochgradig ansteckungsfähigen Patienten vom allgemeinen Verkehr wird also nicht angestrebt. Es entzieht sich meiner Beurteilung, wieweit es möglich ist, im Heeresdienst erkrankte, progredient Lungentuberkulöse in Anstalten, Lazaretten oder Krankenhäusern unterzubringen. Jedenfalls ist der Gedanke, daß diese Kranken in den letzten Wochen ihres Lebens ihre eigenen oder fremde Kinder der ersten Lebensjahre noch infizieren und zugrunderichten, so niederdrückend, daß nach Mitteln und Wegen gesucht werden muß, hier Wandel zu schaffen. Ich bin mir bewußt, daß dabei große Schwierigkeiten bestehen, aber zum mindesten läßt sich auch in diesem Falle erreichen, daß die Kranken bei ihrer Entlassung in die Heimat eine entsprechende Aufklärung über die Natur ihrer Krankheit und die große Gefahr ihrer Uebertragbarkeit auf junge Kinder erhalten. Der Wert dieser Maßnahme wird im einzelnen Fall nicht immer zutagetreten, aber eine Besserung der allgemeinen Tuberkulosemorbidität und -mortalität ist nur $\mathrm{zu}$ erwarten, wenn endlich im Volke die Grundlehren über Aetiologie und Verbreitung der Tuberkulose bekannt werden.

Zusammenfassung. Die Häufung der Kindertuberkulose infolge des Krieges ist neben der einseitigen Kohlehydraternährung zurückzuführen auf eine vermehrte Infektionsgelegenheit mit humanen und bovinen Tuberkelbazillen. Für die humane Infektion ergibt sich eine vermehrte Gelegenheit durch die kürzere oder längere Entfernung vieler Kinder aus dem elterlichen Hausstand, die verschlechterten Wohnungsverhältnisse und die Rückkehr im Heeresdienst an Tuberkulose Erkrankter in die Familie. Für die bovine Infektion ist die verminderte tierärztliche Kontrolle der Viehbestände und das vielfache Fehlen sachkundiger Stallschweizer verantwortlich zu machen. Eine Besserung der bestehenden Verhältnisse ist nur durch eine zielbewußte Prophylaxe zu erreichen. Sie muß vor allem in der Aufklärung aller Lungentuberkulösen über die Art ihrer Erkrankung und die außerordentlich leichte Uebertragbarkeit auf junge Kinder bestehen, es muß ferner eine allgemeine Warnung vor dem Genuß ungekochter Milch erfolgen, und schließlich ist die Zurückhaltung im Felde schwer Erkrankter in Anstalten zu erwägen.

1) Leschke, Zschr. f. Tbe. 27. 1917. 